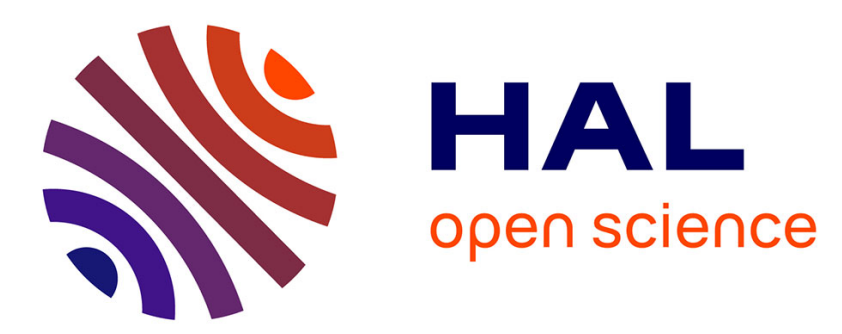

\title{
Design of Molecularly Imprinted Polymeric Materials: The Crucial Choice of Functional Monomers
}

\author{
Amira Anene, Rafik Kalfat, Yves Chevalier, Souhaira Hbaieb
}

\section{To cite this version:}

Amira Anene, Rafik Kalfat, Yves Chevalier, Souhaira Hbaieb. Design of Molecularly Imprinted Polymeric Materials: The Crucial Choice of Functional Monomers. Chemistry Africa, 2020, 3 (3), pp.769781. 10.1007/s42250-020-00180-1 . hal-02994519

\section{HAL Id: hal-02994519 \\ https://hal.science/hal-02994519}

Submitted on 2 Dec 2020

HAL is a multi-disciplinary open access archive for the deposit and dissemination of scientific research documents, whether they are published or not. The documents may come from teaching and research institutions in France or abroad, or from public or private research centers.
L'archive ouverte pluridisciplinaire HAL, est destinée au dépôt et à la diffusion de documents scientifiques de niveau recherche, publiés ou non, émanant des établissements d'enseignement et de recherche français ou étrangers, des laboratoires publics ou privés. 


\title{
Design of molecularly imprinted polymeric materials: The crucial choice of functional monomers
}

\author{
Amira Anene ${ }^{1}$, Rafik Kalfat ${ }^{1}$, Yves Chevalier ${ }^{2}$, Souhaira Hbaieb ${ }^{1,3}$ \\ ${ }^{1}$ Laboratoire Matériaux, Traitement et Analyse, Institut National de Recherche et d'Analyse Physico- \\ Chimique, Biotechpole Sidi-Thabet, 2020 Ariana, Tunisia. \\ ${ }^{2}$ Laboratoire d'Automatique de Génie des Procédés et de Génie Pharmaceutique, UMR 5007 CNRS, \\ Université de Lyon1, 69622 Villeurbanne Cedex, France. \\ ${ }^{3}$ Faculté des Sciences de Tunis, Université de Tunis El Manar, Tunis, Tunisia.
}

\begin{abstract}
:
The choice of the functional monomer is addressed in the present study. It is not only dictated by its ability to interact with the template molecule. Its reactivity towards the cross-linker in the radical polymerization reaction is also to be considered in order to yield a suitable distribution of the monomer bearing binding groups within the material and also to adjust the cross-linking degree which provides rigidity to MIP network. MIPs prepared using two functional monomers of very different reactivity in radical polymerization allowed to investigate the criteria for the optimum choice of the functional monomer. MIPs were made of cross-linked poly(methacrylic acid) or poly(maleic acid) bound as thin films to a silica solid support. Ethylene glycol dimethacrylate was the cross-linker. Calculations of the composition drift of the copolymer material from monomers reactivity ratios give new insights into the control of MIP properties by the choice of the functional monomer. As a consequence of the lower reactivity of maleic acid than methacrylic acid for copolymerization with methacrylic esters, the incorporation of maleic acid is low, the cross-linking density is very high, the polymer coating is very thin, and the specific area is high. The final structure of the MIP network with a predominance of isolated functional units closely surrounded by cross-links yields a rigid material capable of preserving the memory of the model molecule of patulin in molecular imprints. Low reactivity of the functional monomer has beneficial effects regarding the binding selectivity for the target molecule compared to materials prepared from the more reactive methacrylic acid which lead to the formation of flexible polymer formed of short poly(methacrylic acid) sequences.
\end{abstract}

Keywords: Molecular imprinted polymer; reactivity of monomers; reactivity ratios. 


\section{Introduction}

Molecularly imprinted polymers (MIPs) are described as cross-linked polymeric materials containing specific imprints of molecules created during the manufacturing process [1]. MIP materials can establish specific interaction with the target molecules that have been used for the formation of the imprints. The molecular imprints are created by performing the cross-linking reaction (or the full polymerization process including the cross-linking) in the presence of the target molecules. Removal of the target molecules leaves specific imprints for re-binding the target molecule in a specific manner. Crosslinking the polymer ensures the hardening of the MIP and avoids deformation of the formed imprints. Molecularly imprinted polymers (MIPs) are unique materials often referred to as "plastic antibodies" [2], even in cases antigen-antibody interactions are not involved. They are synthesized by in situ polymerization in the presence of template molecules, ions, bio(macro)molecules, or even microorganisms (e.g. viruses). However, this apparently simple paradigm requires the tedious task of monitoring several parameters in view of achieving the dream of a highly selective and sensitive "plastic antibody". MIPs can be used in applications where specific chemical recognition is required: specific electrodes, stationary phase of chromatography, extraction materials and nanomedicine $[1,3-5]$. It is mandatory that cross-linking of the polymer material retains the structural features of the interaction site between the MIP and the target molecule after the later has been removed. High contents of cross-linking agent are used for that purpose.

There is a need for a high enough amount of functional monomer to ensure a high binding capacity for the target molecule and much cross-linking agent for the material hardening. Functional monomers must interact with the target molecule in order to form a prepolymerization complex (PPC) which is essential for the formation of specific binding 
sites [6]. The use of an adequate proportion of monomer/template in the prepolymerization mixture leads to the formation of a large number of high-affinity sites. Several theoretical studies [7, 8], have been done as tools for the selection of the optimum monomer-template ratio. To this end, computer simulations were used to estimate the monomer-template interaction energies. However, caution should be taken in their direct application to real systems; the most suitable estimation which takes into account the complexity of the real medium must be empirically derived [9]. Besides the interaction between the functional monomer and the template, the functional monomer should also be chosen on the basis of its reactivity in the radical copolymerization with the crosslinking agent. Indeed, it has been recognized early that both vinyl monomers have different reactivities, which leads to a of chemical composition drift of the copolymer during the course of the copolymerization [see Ref. [1], pp. 27-32]: the most reactive monomer is consumed at the beginning of copolymerization, so that the first formed macromolecules are rich with the most reactive monomer. The less reactive monomer polymerizes at the end when the most reactive one has been consumed, so that the copolymers produced at the end are rich with the less reactive monomer. As far as we know, though these facts have been acknowledged, their consequences on the properties of MIPs have not been considered so far. This is the purpose of the present work [9]. The present work aims at investigating show, how relevant are the respective reactivities of the functional monomer and the cross-linking agent and their effect on the final structure of the imprinted polymer. This is achieved via a systematic study based on kinetic appraisal of MIP chemical composition and morphological characterization of the resulting materials. Besides it provides simple guidelines for the selection of functional monomer and cross-linking agent, this contribution gives a rationale for the low 
adsorption capacity of highly selective adsorbents based on molecular imprinted polymers formed by radical polymerization.

The present molecularly imprinted polymer materials taken as a case study are thin films of cross-linked poly(carboxylic acid)s bound to the surface of silica gel [10]. The pair of co-monomers was ethylene glycol dimethacrylate (EGDMA) as a cross-linking agent and either methacrylic acid (MAA) or maleic acid (MA) as the functional monomer. Such materials have been designed for the specific binding of patulin (PAT) [11], a toxic mycotoxin produced by filamentous fungi, Penicillium, Aspergillus and Byssochlamys $[12,13]$, possibly contaminating fruits and vegetables [14]. The MIPs were denoted as Sil-MAA/MIP and Sil-MA/MIP respectively for MAA or MA used as a monomer. Nonimprinted polymers prepared in the same manner than MIPs but in the absence of patulin; they are abbreviated/defined as Sil-MAA/NIP and Sil-MA/NIP.

\section{Materials and Methods}

\section{Materials}

Methacrylic acid (MAA), maleic acid (MA), ethylene glycol dimethacrylate (EGDMA) (see Scheme 1 for chemical structures) 2,2'-azobisisobutyronitrile (AIBN) were purchased from Sigma-Aldrich. Patulin from A.G. Scientific (San Diego, CA), was a standard of purity $\geq 98 \%$. The silica support bearing grafted with methacryloyl groups at its surface (Sil- $\gamma$-MPTS) was prepared by the Stöber sol-gel process from tetraethyl orthosilicate and $\gamma$-methacryloxypropy ltrimethoxysilane as reported in a previous paper [10]. Deionized water was filtered with $0.45 \mu \mathrm{m}$ Nylon membrane filter before use. 


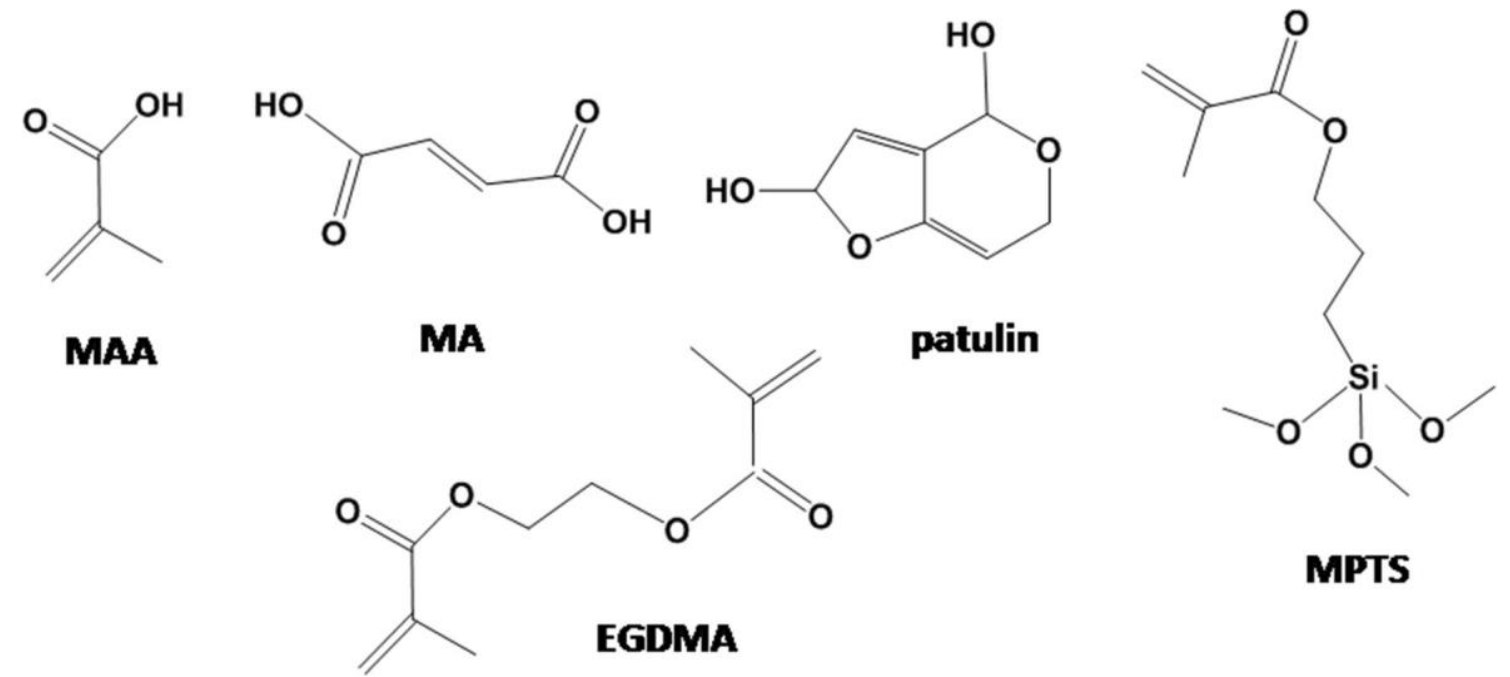

Scheme 1. Chemical structures of reactants: methacrylic acid (MAA), maleic acid (MA), ethylene glycol dimethacrylate (EGDMA), 3-methacryloxypropyltrimethoxysilane (MPTS).

\section{Preparation of MIP Material}

The molecularly imprinted polymer materials attached as thin films on the silica solid support were prepared as previously described [10]. The silica support Sil- $\gamma$-MPTS and $0.01 \mathrm{mmol}$ of Patulin template were dispersed by ultrasounds for $10 \mathrm{~min}$ in $1 \mathrm{~mL}$ of acetonitrile. $20 \mathrm{mmol}$ of cross-linker EGDMA and either $20 \mathrm{mmol}$ of methacrylic acid or maleic acid monomer and the AIBN initiator $(0.2 \mathrm{mmol})$ were added into the solution. The polymerization vessel was deoxygenated by bubbling nitrogen gas for $10 \mathrm{~min}$. Polymerization was performed by heating at $60{ }^{\circ} \mathrm{C}$ for $6 \mathrm{~h}$. The solid powder was filtered and thoroughly washed with acetonitrile for removing all species that have not been attached to the silica support. Removal of patulin was performed by several washing cycles in a Soxhlet extractor during $8 \mathrm{~h}$ with ethyl acetate, followed by $8 \mathrm{~h}$ with methanolacetic acid (90/10) mixture. Completion of patulin extraction from the accessible surface sites was checked by HPLC analyses for the extracted patulin in the solvent [15]. 


\section{Methods}

\section{Analyses of the grafted polymer content}

Elemental analyses for Carbon and Hydrogen were performed on a Perkin-Elmer 2400 series II instrument. ThermoGravimetric Analysis (TGA) measurements were carried out on a TG 209 F1 Netzsch instrument. The samples were weighed in alumina crucibles inserted in a tubular oven heated by a nitrogen gas flow from $20^{\circ} \mathrm{C}$ to $900{ }^{\circ} \mathrm{C}$ at a rate of $10^{\circ} \mathrm{C} \cdot \mathrm{min}^{-1}$.

\section{Morphology characterizations}

Specific area and porosity were assessed by measurements of BET adsorption isotherms of nitrogen gas at $77 \mathrm{~K}$. Adsorption and desorption isotherm of nitrogen gas were measured using a Micromeritics Tristar3000 V6.04 BET instrument. The type of adsorption isotherm was inferred from the full data measured between 0.05 to 0.3 relative pressures. The specific area was calculated from a fit of the BET equation to the adsorption isotherm in the relative pressure range $p / p_{0}$ from 0.05 to 0.3 and the pore volume was analyzed from the desorption branch by the Barrett-Joyner-Halenda (BJH) method.

Transmission Electron Microscopy (TEM) was performed at the 'Centre Technologique des Microstructures' facility (University of Lyon; http://microscopies.univ-lyon1.fr) on a Philips CM120 microscope operating at $80 \mathrm{kV}$ acceleration. A dilute aqueous suspension $(0.1 \%)$ of powder materials was spread on Formvar/carbon grids and dried before observation.

\section{Adsorption experiments}

Adsorption isotherms were measured by the depletion method. $30 \mathrm{mg}$ of MIP or NIP materials were equilibrated overnight with $1.0 \mathrm{~mL}$ of $5 \mu \mathrm{g} \cdot \mathrm{mL}^{-1}$ patulin solution. The 
liquid medium was collected by centrifugation at $5000 \mathrm{rpm}$ for $10 \mathrm{~min}$ and analyzed for patulin by HPLC. The adsorbed amount, $\Gamma\left(\mu \mathrm{mol} \cdot \mathrm{g}^{-1}\right)$, was calculated from:

$$
\Gamma=\frac{\left(C_{0}-C\right) V}{m}
$$

where $C_{0}$ was the total concentration of patulin, $C$ was the equilibrium concentration of patulin measured in the liquid medium, $V$ was the volume of the patulin solution and $m$ was the mass of the material.

\section{Results and Discussion}

\section{Preparation of molecularly imprinted polymeric materials}

The radical copolymerization of the functional monomer (methacrylic acid or maleic acid) and the EGDMA cross-linker was operated in the suspension of silica solid particles bearing surface bound methacryloyl groups. These latter ensure the attachment of the polymer material developed through a grafting from process. However, only the part of the polymer that incorporates the grafted methacryloyl groups is attached. A large part of the polymer is present in solution or as microgel particles suspended in acetonitrile. Such non-attached materials are eliminated during the filtration and washing of the solid powder. Methacrylate monomers are highly reactive in radical polymerization so that copolymerization of the grafted methacryloyl groups reached completion. Indeed infrared spectroscopy analyses do not reveal residual vinyl groups at $1630 \mathrm{~cm}^{-1}$ in the final materials. It is worth considering the particular behavior of the grafted methacryloyl groups: they are highly reactive and close together on the silica surface so that their copolymerization is fast. Since their amount is lower than the amounts of soluble functional monomer and cross-linking agent, the copolymerization of the grafted methacryloyl groups takes place during the early times of the copolymerization. Once 
polymerization of the grafted methacryloyl groups is completed, only non-attached materials can form upon further polymerization of the residual soluble monomers. The chemical compositions of the grafted and non-grafted copolymers are different because of the composition drift of the copolymers along polymerization time coming from the different reactivities of the monomers. The copolymerization taking place at the silica surface is efficient as the mass of solid materials significantly increased after polymerization and infrared spectroscopy analyses reveal large amounts of polymer in the coated silica materials. The mass fraction of polymer is deduced from elemental and thermogravimetric analyses reported in the next section. The final products are in the form of a solid powder which surface is not sticky and do not swell with water, showing that cross-linking of the grafted layer is high. Scheme 2 illustrates the preparation steps of the molecularly imprinted polymeric materials.

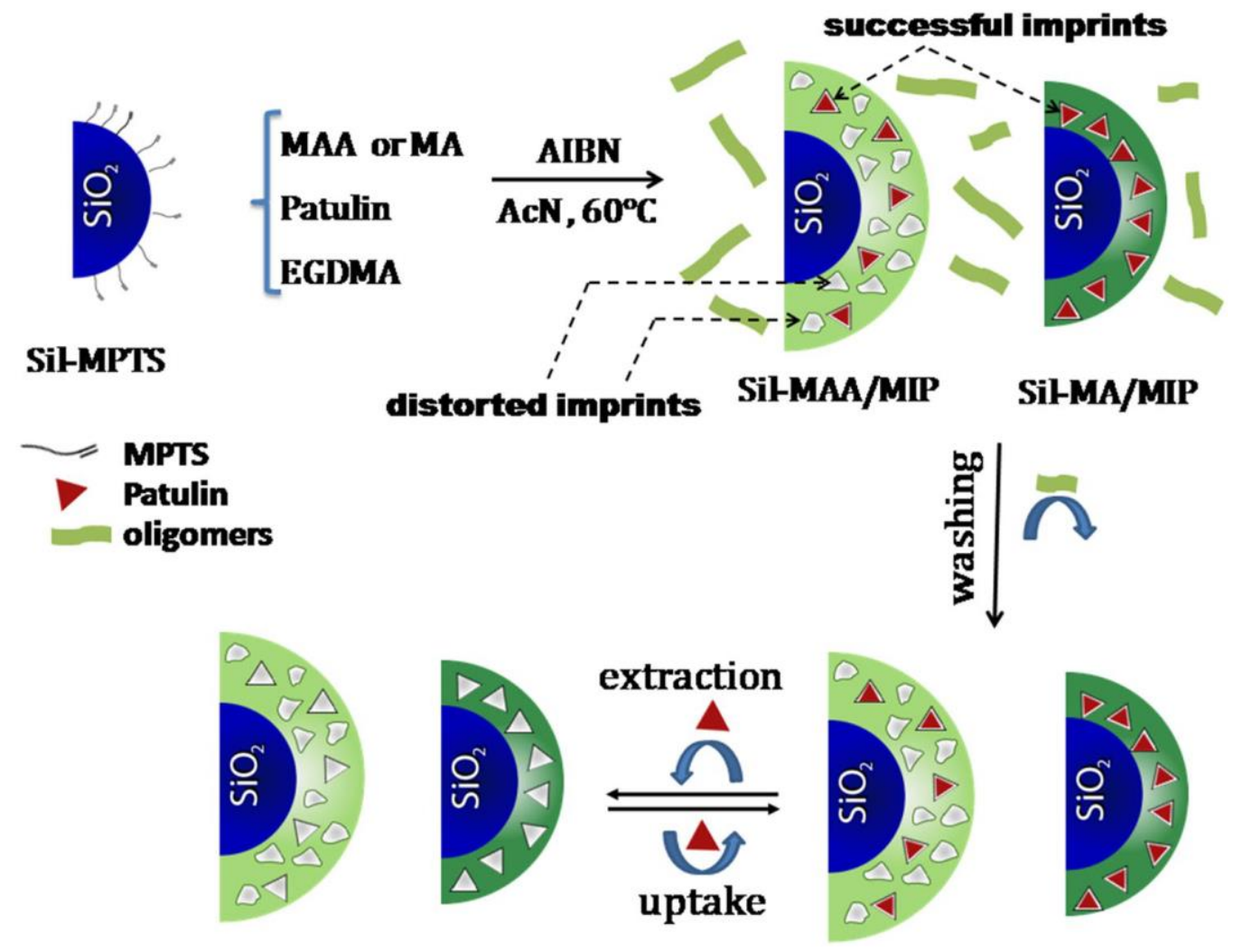

Scheme 2. Schematic illustration of Sil-MIP preparation steps. 


\section{Chemical characterization of the materials}

The amount of polymer materials attached to the silica surface is inferred from elemental analyses of $\mathrm{C}$ and $\mathrm{H}$ and thermogravimetric analyses (Table 1). Elemental analysis of carbon of Sil- $\gamma$-MPTS reveals the high density of grafted methacryloyl groups at the surface of silica. $9.0 \mathrm{wt} \%$ carbon content corresponds to a surface density of $1.07 \mathrm{mmol} \cdot \mathrm{g}^{-1}$ or $3.1 \mu \mathrm{mol} \cdot \mathrm{m}^{-2}$ assuming a specific area of silica particles of $350 \mathrm{~m}^{2} \cdot \mathrm{g}^{-1}$ as determined by BET. Such density is in the right range for a dense coverage as a monolayer where each $\gamma$-MPTS occupies a mean area of $0.54 \mathrm{~nm}^{2}$. The methacryloyl groups are close-packed at the silica surface; the mean center-to-center distance between them is $0.8 \mathrm{~nm}$, close to the diameter of $0.9 \mathrm{~nm}$ for a $\gamma$-MPTS molecule calculated assuming its spherical shape. The carbon contents of Sil-MAA/MIP and Sil-MA/MIP materials are much higher than that of Sil- $\gamma$-MPTS as a consequence of copolymerization involving the surface-bound methacryloyl groups. The amount of polymer material is $\sim 50 \%$ higher for Sil-MAA/MIP than Sil-MA/MIP.

Table 1. Grafting density deduced from elemental and thermogravimetric analyses.

\begin{tabular}{|l|l|l|l|l|}
\hline Material & $C(\mathrm{wt} \%)$ & $\begin{array}{l}\text { Mass loss } \\
(\mathrm{wt} \%)\end{array}$ & $\begin{array}{l}\text { Corrected mass } \\
\text { loss }(\mathrm{wt} \%)\end{array}$ & $\begin{array}{l}\text { Grafting density } \\
(\mathrm{mmol} \mathrm{g}-1)\end{array}$ \\
\hline Sil-MPTS & 9.0 & 21 & 14 & 1.1 \\
Sil-MAA/MIP & 47.1 & 78 & 50 & 2.3 \\
Sil-MA/MIP & 32.9 & 75 & 47 & 1.4 \\
\hline
\end{tabular}

Thermogravimetric analyses (TGA) are presented in Figure 1. Water loss coming from dehydration of the surface silanol of silica takes place concomitantly with the thermal degradation. The mass loss of Sil- $\gamma$-MPTS corresponding to the organic grafts is estimated as the height of the step between the tangents beyond and above the temperature range of thermal degradation (from $350{ }^{\circ} \mathrm{C}$ to $500{ }^{\circ} \mathrm{C}$ ). The mass loss of $21 \%$ is corrected to $14 \%$ after subtraction of the water release. This corresponded to a surface density of $1.1 \mathrm{mmol} \cdot \mathrm{g}^{-1}$ taking the molar mass of the grafted moiety as $127 \mathrm{~g} \cdot \mathrm{mol}^{-1}$, which is 
identical to that determined from elemental analysis of carbon. The mass losses for SilMAA/MIP and Sil-MA/MIP are 78 and $75 \%$, respectively, which are quite high values. Surprisingly, there is no mass loss between room temperature and the onset of thermal degradation of the grafts although the same dehydration of surface silanols should have taken place. It is presumed that the dense coating with the polymer layer prevented water loss until the organic coating has been degraded. The correction for water release cannot be done in the same way as for Sil- $\gamma$-MPTS. The full water loss corresponding to dehydration of silica from room temperature to the end of thermal degradation at $450{ }^{\circ} \mathrm{C}$ was subtracted from the overall mass loss, which gives a correction of $28 \mathrm{wt} \%$.

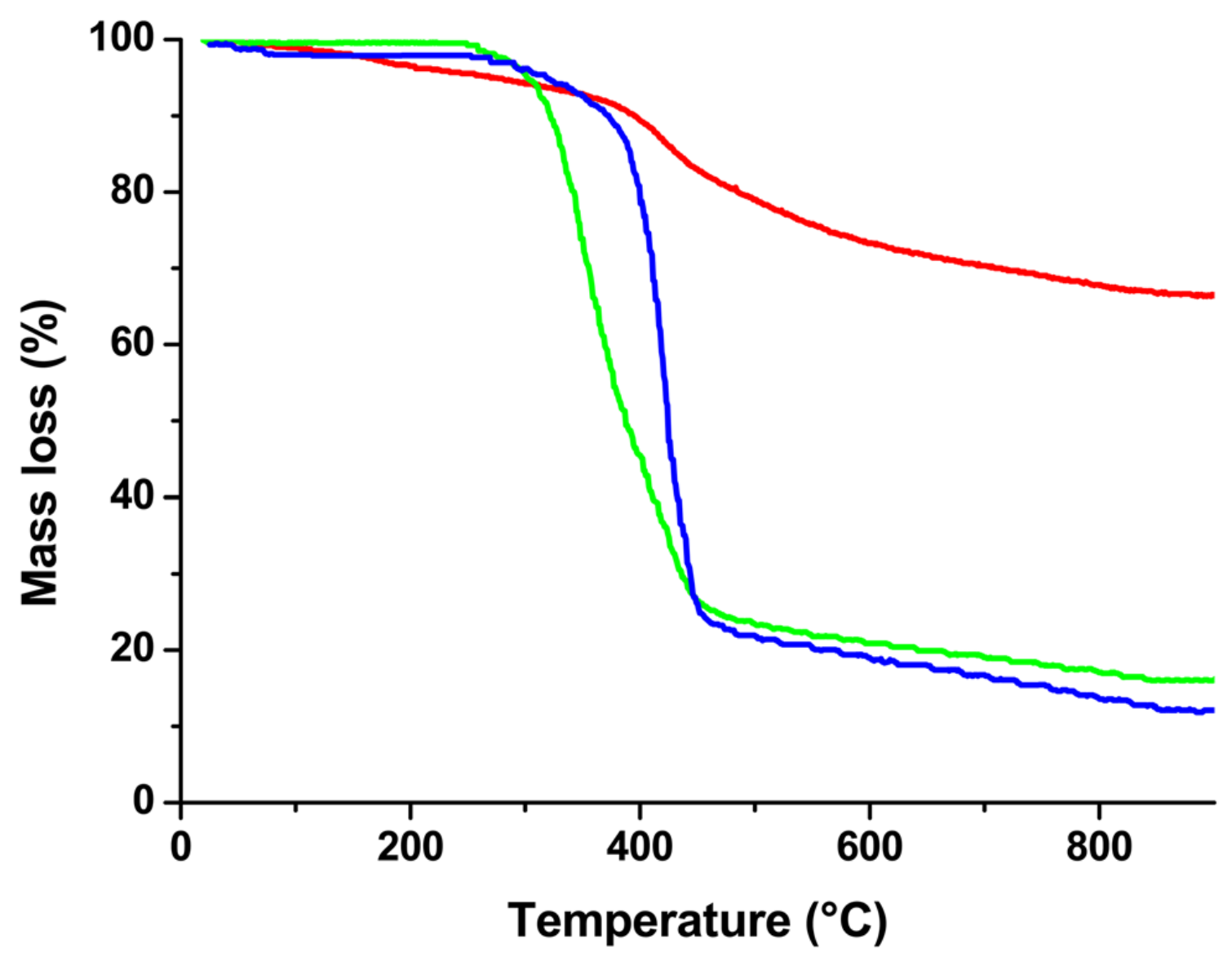

Figure 1. TGA analysis of Sil-MPTS (red), Sil-MAA/MIP (blue) and Sil-MA/MIP (green). 
Elemental analysis provides a much better accuracy because the mass loss measured in TGA is continuous and includes a progressive water loss coming from dehydration of the surface silanol of silica. The deduction of the mass loss from TGA curve related to the degradation of the grafted moieties not including that corresponding to water loss is very sensitive to the choice of the temperature range where the mass loss is taken and the correction for water release in this temperature range.

The conversion of carbon analysis or TGA mass loss into moles of grafted polymer requires the knowledge of the chemical structure of the polymers. Such conversion can be done for Sil- $\gamma$-MPTS because the chemical formula of the grafts is known. A tentative conversion for the polymers is done assuming that the chemical composition of the copolymers is the same as the monomer load in the polymerization vessel, that is, $1: 1$ mole ratio. The grafted densities calculated in that way from elemental analysis and TGA respectively are $2.3 \mathrm{mmol} \cdot \mathrm{g}^{-1}\left(26.7 \mu \mathrm{mol} \cdot \mathrm{m}^{-2}\right)$ and $1.8 \mathrm{mmol} \cdot \mathrm{g}^{-1}\left(20.9 \mu \mathrm{mol} \cdot \mathrm{m}^{-2}\right)$ for SilMAA/MIP and $1.4 \mathrm{mmol} \cdot \mathrm{g}^{-1}\left(4.1 \mu \mathrm{mol} \cdot \mathrm{m}^{-2}\right)$ and $1.5 \mathrm{mmol} \cdot \mathrm{g}^{-1}\left(4.4 \mu \mathrm{mol} \cdot \mathrm{m}^{-2}\right)$ for SilMA/MIP. Such grafted densities are of the same order of magnitude from elemental analyses and TGA; there are small but significant differences between the two methods, however. The values from elemental analysis are considered more reliable but the conversion into grafted densities in $\mathrm{mmol} \cdot \mathrm{g}^{-1}$ remained linked on a hypothesis regarding the chemical composition of the grafted copolymers.

The higher grafted polymer content in Sil-MAA/MIP than Sil-MA/MIP can be directly ascribed to the higher reactivity of methacrylic acid compared to maleic acid in the radical polymerization processes. This first view is strictly valid for a homopolymerization; it requires being discussed deeper into details because a copolymerization with EGDMA is here taking place. 


\section{Structural characterization of the materials}

The morphology of the polymer coatings at the surface of the silica solid support is assessed by means of TEM pictures and nitrogen gas adsorption experiments. The BET adsorption isotherms (Figure 2) recorded between 0.005 and 0.99 relative pressure $p / p_{0}$ displayed monotonous increases of adsorbed volume with respect to $p / p_{0}$, the adsorption towards low relative pressures looked reaching the origin, and they are close to reversibility as they did not show a large hysteresis between the adsorption and desorption branches (Figure 2). This matched the type II isotherm corresponding to non-porous materials according to the classification of IUPAC [16]. The specific areas are determined by fitting the BET equation to the experimental adsorption isotherms. The specific area and the $c$ interaction energy parameter of the BET theory are given in Table 2. The specific areas are high because the sol-gel synthesis process of silica yields a porous material made of small elementary nanoparticles irreversibly sintered as porous aggregates. The specific areas of Sil-MA/NIP and Sil-MA/MIP above $200 \mathrm{~m}^{2} \cdot \mathrm{g}^{-1}$ are close to that of the starting silica. The difference between those two samples probably comes from small differences in the sol-gel synthesis conditions. The striking feature is the much smaller specific area of Sil-MA/MIP that revealed a loss of specific area upon copolymerization of MAA and EGDMA onto Sil- $\gamma$-MPTS. Such a trend suggested that the polymer coating encapsulated several elementary silica particles, thus filling the interstices in between them and removing part of the original specific area of the Sil- $\gamma$ MPTS silica. Under such a phenomenon, the porous silica material made by a sol-gel synthesis has been converted into larger less-porous particles. 


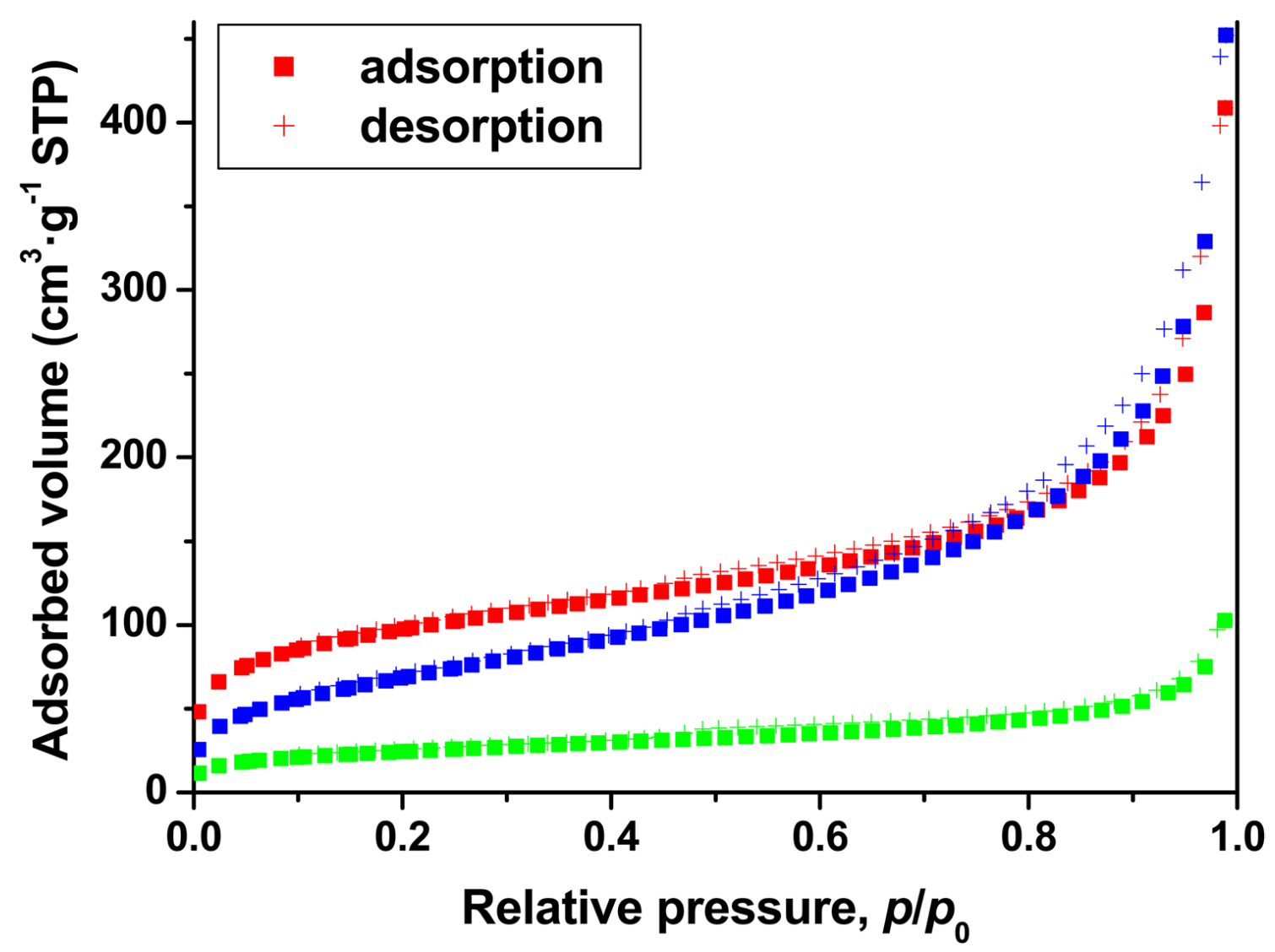

Figure 2. BET adsorption (filled square) and desorption (+) isotherms of Sil-MA/MIP (red), Sil-MA/NIP (blue) and Sil-MAA/MIP (green).

Table 2. Specific area and interaction energy parameter $c$, extracted from BET measurements.

\begin{tabular}{|c|c|c|}
\hline Material & $\begin{array}{c}\text { Specific area } \\
\left(\mathrm{m}^{2} \mathrm{~g}^{-1}\right)\end{array}$ & $c$ \\
\hline Sil-MA/NIP & 255 & 57 \\
Sil-MA/MIP & 344 & 220 \\
Sil-MAA/MIP & 86 & 180 \\
\hline
\end{tabular}

Transmission electron microscopy pictures of the MIP materials based on copolymerization of MAA or MA with EGDMA reveals large differences of morphologies (Figure 3). The pictures at moderate and high magnifications of SilMA/MIP show that the silica particles are encapsulated by an MA-EGDMA copolymer layer that keeps the porous morphology of the starting Sil- $\gamma$-MPTS sol-gel silica. On the contrary, the pictures of Sil-MAA/MIP show the strong aggregation of the material as the MAA-EGDMA copolymer encapsulated several Sil- $\gamma$-MPTS particles inside a single 
dense aggregate. The pictures of Sil-MAA/MIP also show parts of material made of pure copolymer appearing as materials of low electron density with a smooth surface. These observations provide a visual confirmation of the conclusion drawn from the loss of BET specific area induced by the polymerization yielding the Sil-MAA/MIP material. Indeed, chemical analyses of Sil-MAA/MIP have shown that polymerization yielded a larger amount of grafted copolymer than for Sil-MA/MIP.
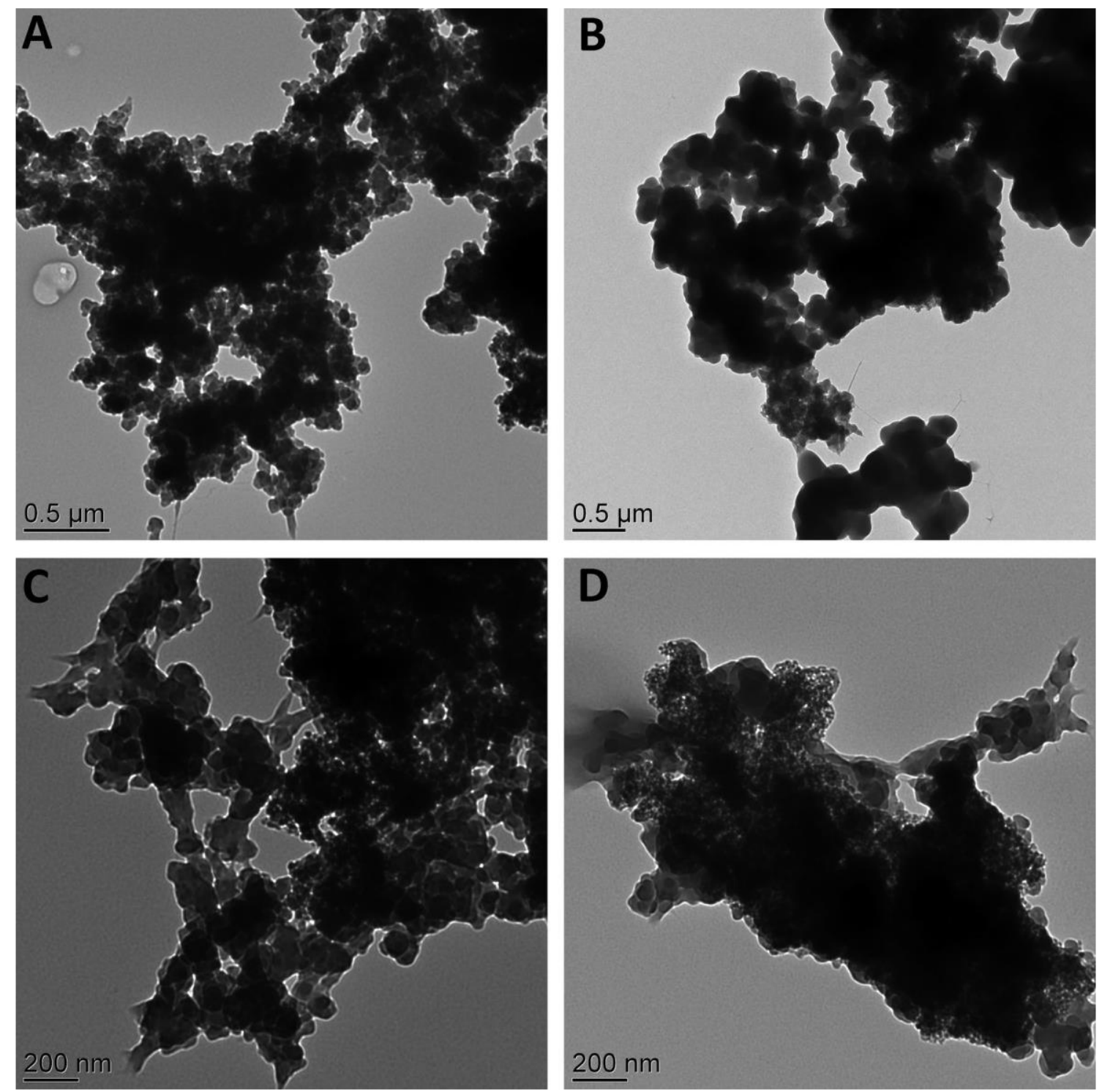

Figure 3. TEM pictures of Sil-MA/MIP (a, c) and Sil-MAA/MIP (b, d). 


\section{Adsorption of patulin on MIPs and NIPs}

As reported in a previous paper, the equilibrium adsorption of patulin from a $5 \mu \mathrm{g} \cdot \mathrm{mL}^{-1}$ $\left(32 \mu \mathrm{mol} \cdot \mathrm{L}^{-1}\right)$ aqueous solution is $\Gamma=1.62 \mu \mathrm{mol} \cdot \mathrm{g}^{-1}$ for $\mathrm{Sil}-\mathrm{MA} / \mathrm{MIP}$ and $\Gamma=1.15 \mu \mathrm{mol} \cdot \mathrm{g}^{-1}$ for Sil-MAA/MIP. Adsorption to the NIPs reaches the same value of $\Gamma=0.52 \mu \mathrm{mol} \cdot \mathrm{g}^{-1}$ for both Sil-MA/NIP and Sil-MAA/NIP. From these values, a imprinting factor is calculated as $\alpha=\frac{\Gamma_{\mathrm{MIP}}}{\Gamma_{\mathrm{NIP}}}$, yielding $\alpha=3.1$ for Sil-MA/MIP and $\alpha=2.2$ for Sil-MAA/MIP. The two important outcomes are a higher selective binding to molecular imprints for Sil-MA/MIP than Sil-MAA/MIP and identical non-selective adsorption to Sil-MAA/NIP and Sil-MAA/NIP. In other words, the materials based on either MA or MAA were identical for the non-selective adsorption showing that interactions between patulin and the different copolymer surfaces are identical. Those interactions are predominantly physical interactions (van der Waals) and acid-base (hydrogen bonding) interactions involving carboxylic acid groups of the polymer that have not been oriented by the presence of patulin during the copolymerization process. The selective contribution to the adsorption is higher for Sil-MA/MIP, suggesting that it has a higher surface density of molecular imprints. Although the Sil-MAA/MIP contains more imprints due to the higher amount of polymer compared to Sil-MA/MIP, the selective binding is less. This means that the formation of molecular imprints largely failed during the preparation process of Sil-MAA/MIP. As the successful formation of molecular imprints requires an efficient quench of the materials around the template molecules through cross-linking, it is concluded that the Sil-MA/MIP material is made more rigid because of its higher degree of cross-linking. Computational chemistry may help at providing better understanding of the higher uptake of patulin by Sil-MA/MIP compared to Sil-MAA/MIP [17]. 


\section{General Discussion}

There have been extensive studies for the choice of the starting materials and synthesis routes leading to the preparation of MIPs $[6,18,19]$. The functional monomers should strongly interact with the template molecules, but other parameters also matter $[20,21]$. Such interactions depend also on the liquid medium used during the copolymerization. The solvent for the monomers and the template should not interfere too much, but it should be able to dissolve all the starting ingredients so that it necessarily operates interactions with them. Acetonitrile was used in the present study as it has been extensively used for MIPs based on poly(carboxylic acids) [22, 23]; this is a basic solvent able to interact with the carboxylic acids of the functional monomers and the alcohol groups of patulin. The solvent is also used as a "porogen" in case of precipitation polymerization aiming at the manufacture of polymer microparticles (beads). Indeed, a large binding capacity requires a large specific area, therefore porous microparticles. In the present case, the copolymerization was performed at the surface of porous solid support made of silica, so that it is not useful that the solvent acts as a porogen.

The present discussion focuses on the selection of the functional monomer and its reactivity in radical copolymerization. The two monomers differ by their number of acidic groups and their acidity, and by their reactivity in the radical copolymerization reaction with EGDMA.

The two MAA-co-EGDMA and MA-co-EGDMA copolymers have carboxylic acid groups of different acidities. It is difficult to give definite values of $\mathrm{p} K_{\mathrm{A}}$ for polymer materials because the acidity of the carboxylic groups along the polymer chain varies as a function of the degree of neutralization [24] and both the nature and the distribution of the comonomer [25-28]. However, taking the values at half neutralization as typical $\mathrm{p} K_{\mathrm{A}}$, orders of magnitudes are $\mathrm{p} K_{\mathrm{A}} \approx 7-8$ for a random $1: 2$ copolymer of methacrylic acid and 
ethyl acrylate in aqueous solution [29], and $\mathrm{p} K_{\mathrm{A} 1} \approx 4.5$ and $\mathrm{p} K_{\mathrm{A} 2} \approx 9.5$ for an alternate copolymer of maleic acid and isobutene [28]. Though, the maleic acid units of MA-coEGDMA copolymers bear two acidic groups, one of them is quite acidic but the second one is a very weak acid, so that the maleic acid units finally behave as monocarboxylic acids in the same way as the methacrylic acid units of MA-co-EGDMA copolymers. Therefore, the Sil-MA/MIP and Sil-MAA/MIP have the same number of operative acidic groups per functional unit, the MA units are more acidic than the MAA units, and the amount of grafted copolymer is larger for Sil-MAA/MIP.

The first intuitive consequence of the higher reactivity of MAA than MA is binding a larger amount of copolymer to the silica surface by means of copolymerization with the grafted methacryloyl groups. Indeed, a higher reactivity manifested by a larger propagation rate constant in radical polymerization might afford macromolecules of larger molar masses. For a homopolymerization in homogeneous solution with no radical transfer reaction, the kinetic length of a polymer chain, $\lambda$, results from the competition between propagation rate of the polymerization (chain growth) and termination reactions (by any chemical mechanism):

$\lambda=\frac{k_{\mathrm{p}}[\mathrm{M}]}{2\left(f k_{\mathrm{d}} k_{\mathrm{t}}[\mathrm{I}]\right)^{1 / 2}}$

where $k_{\mathrm{p}}, k_{\mathrm{d}}$ and $k_{\mathrm{t}}$ are the rate constants for propagation, thermal decomposition of the initiator and termination reactions, $f$ is the efficiency factor of the initiation reaction, [M] and $[\mathrm{I}]$ are the concentrations of monomer and initiator (AIBN). The final mean polymerization degree is either $\lambda$ for a termination by radical dismutation or $2 \lambda$ for a termination by radical coupling. Indeed, high reactivity of the monomer should lead to high $k_{\mathrm{p}}$ and high molar mass. But high reactivity can also lead to high initiation rate $\left(=2 f k_{\mathrm{d}}[\mathrm{I}]\right)$ and high termination rate of the growing macroradicals $\mathrm{M}_{\mathrm{n}}{ }^{\bullet}\left(=k_{\mathrm{t}}\left[\mathrm{M}_{\mathrm{n}}{ }^{\circ}\right]^{2}\right)$, 
so that the effects of high reactivity on the rates of all reactions might compensate for one another.

Considering the details of copolymerization kinetics allows inferring the chemical composition of the MIP material grafted on the silica support based on the same theoretical approach as for copolymerization in homogeneous solution. Firstly, the copolymerization taking place at the surface incorporates the grafted methacryloyl monomers in the macromolecules and the amount of grafted polymer stops growing when the full grafted methacryloyl monomers have been converted. Further polymerization yields polymer materials that are not attached to the silica support; they will be eliminated during subsequent washing processes. High molar mass macromolecules incorporate a larger number of grafted monomer so that the full conversion of grafted methacryloyl monomers is reached earlier and the amount of grafted polymer material is finally better represented by the chemical composition of the grafted copolymer (the ratio of the surface methacryloyl to the soluble monomer) than the molar mass of the polymer. Secondly, copolymerization with the cross-linking agent yields a copolymer containing both the functional monomer and the cross-link units that are not operative in binding the target molecules. The parameter that matters most is the amount of bound functional monomer units, expressed in the chemical composition of the copolymers. This chemical composition depends on the reactivity ratio of the monomers. The various monomers are: - grafted methacryloxypropyl groups that should have the same reactivity in copolymerization as methacryloyl ester such as the well-documented butyl methacrylate; - ethylene glycol dimethacrylate (EGDMA) that is also an ester of methacrylic acid of reactivity close to butyl methacrylate;

- the functional monomer, either methacrylic acid or maleic acid. 
As a whole, the copolymerization of a 1:1 mole ratio mixture of functional monomer and both EGDMA and grafted methacryloyl looks like the copolymerization of the functional monomer and other monomers reacting in the same way as butyl methacrylate. For 1 mole of functional monomer, two vinyl groups are coming from EGDMA and an unknown number of surface-bonded methacryloyl monomers coming from the grafted layer of high surface density. Though the mole ratio of different vinyl groups is difficult to assess with confidence, it is clear that the mole ratio of functional monomer is low. This is a good point with regards to the selective binding of the target molecule; but this is at the expense of its binding capacity. It has been claimed that a high amount of cross-linking agent (X) and a low amount of template molecule $(\mathrm{T})$ relative to the functional monomer $(\mathrm{M})$, are necessary so that a high selectivity of binding can be reached [22]. Systematic investigations coupled with experimental design indeed confirmed these trends. Thus, in the case of poly(methacrylic acid) cross-linked using EGDMA, an optimum for selective capacity for binding sulfamethazine was determined as T:M:X $=1: 10: 55$; such optimum ratio was 1:10:10 for less selective binding of five mixed sulfonamides [22]. Another study concluded for an optimum $\mathrm{M}$ :X ratio of 1:1 for binding bisphenol-A to poly(4-vinyl pyridine) cross-linked using either EGDMA or the trifunctional trimethylol propane trimethacrylate [30]. It appears indeed that a mole content of cross-linker at least larger than that of the monomer $(X: M \geq 1: 1)$ is a definite requirement. The exact optimum value is specific to each particular case. The optimum also depends on the choice of the parameter(s) to be optimized: either the binding capacity or the selectivity of binding; also depends on the way selectivity is defined. In the present case, selectivity was estimated by the binding capacity of the MIP compared to that of the NIP. In other studies, selectivity was defined from the competitive binding of the target molecule and 
interfering molecules of similar chemical structure with no reference to binding to the NIP.

The chemical composition of the grafted polymer layer depends on the reactivities in the copolymerization reaction; it is different from that of the M:X ratio. The capacities for selective binding of patulin to Sil-MA/MIP and Sil-MAA/MIP determined as the difference between MIP and NIP are related to this chemical composition. Polymerization of each co-monomer $\mathrm{M}_{1}$ and $\mathrm{M}_{2}$ takes place by their reactions to the growing macroradicals terminated either by $\mathrm{M}_{1}$ or $\mathrm{M}_{2}$.

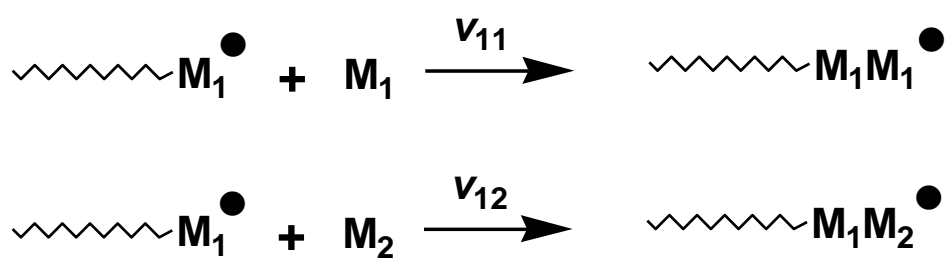

The rates of the reactions are established as follows:

$v_{11}=k_{11}\left[\mathrm{M}_{1}^{\bullet}\right]\left[\mathrm{M}_{1}\right]$

$v_{12}=k_{12}\left[\mathrm{M}_{1}^{\bullet}\right]\left[\mathrm{M}_{2}\right]$

The reactivity ratio can be defined as: $\quad r_{12}=\frac{k_{11}}{k_{12}}$

The same types of equations hold for the reactivity ratio $r_{21}$. When $r_{12}>1$, the macroradical $\mathrm{M}_{1}{ }^{\circ}$ reacts faster with the monomer $\mathrm{M}_{1}$ than $\mathrm{M}_{2}$, so that homopolymerization of $\mathrm{M}_{1}$ is favored. On the contrary $r_{12}<1$, means that the macroradical $\mathrm{M}_{1}{ }^{\cdot}$ reacts faster with the monomer $\mathrm{M}_{2}$, so that copolymerization takes place. Random copolymerization of $\mathrm{M}_{1}$ and $\mathrm{M}_{2}$ requires that both $r_{12}$ and $r_{21}$ are close to 1. An alternate copolymer $\left(\ldots \mathrm{M}_{1} \mathrm{M}_{2} \mathrm{M}_{1} \mathrm{M}_{2} \mathrm{M}_{1} \mathrm{M}_{2} \ldots\right)$ forms when $r_{12}<1$ and $r_{21}<1$. Such reactivity ratios are given in databases for most common monomer pairs [31]. Taking the data of butyl methacrylate (BuMA) as representing both EGDMA and the grafted methacryloyl, together with that of methacrylic acid and maleic acid, the full reactivity data is presented in Table 3. The reactivity ratios of the MA-BuMA pair are not available 
in databases. Therefore they were calculated from the reactivity ratios of the MA-Styrene pair that have been experimentally determined by Świtala-Żeliazkow [32] using the Alfrey-Price $Q$ and $e$ parameters of MA calculated from the reactivity ratios: $Q_{\mathrm{MA}}=0.34$ and $e_{\mathrm{MA}}=1.14$ [32]. From these values together with the values for BuMA taken from databases: $Q_{\mathrm{BuMA}}=0.82$ and $e_{\mathrm{BuMA}}=0.28$ [33], the reactivity ratios of the MA-BuMA pair were estimated (Table 3 ) as

$r_{\mathrm{MA}-\mathrm{BuMA}}=\frac{Q_{\mathrm{MA}}}{Q_{\mathrm{BuMA}}} e^{-e_{\mathrm{MA}}\left(e_{\mathrm{MA}}-e_{\mathrm{BuMA}}\right)}$
$r_{\mathrm{BuMA}-\mathrm{MA}}=\frac{Q_{\mathrm{BuMA}}}{Q_{\mathrm{MA}}} e^{-e_{\mathrm{BuMA}}\left(e_{\mathrm{BuMA}}-e_{\mathrm{MA}}\right)}$

Table 3. Reactivity ratios of the monomer pairs of present relevance.

\begin{tabular}{|c|c|c|c|c|}
\hline $\mathrm{M}_{1}$ & $\mathrm{M}_{2}$ & $r_{12}$ & $r_{21}$ & References \\
\hline MAA & BuMA & 0.75 & 1.20 & {$[34]$} \\
MA & BuMA & 0.16 & 3.07 & See text \\
\hline
\end{tabular}

Useful information on the behavior of the monomer during the polymerization process, the chemical composition of the polymer layer and the cross-linking density of the polymer materials can be inferred from the reactivity ratios for the MA and MAA functional monomers. It is worth noticing that the grafted methacryloyl monomers were highly concentrated at the surface of the silica particles, so that they were rapidly converted into copolymer once a radical reached the surface. The grafted copolymers were formed at the beginning of the polymerization till the full grafted methacryloyl monomers were converted; thereafter the copolymers formed at the end of the polymerization were not attached to the surface and were eliminated upon washing. The reactivity ratios for the MAA-BuMA pair are rather close to one, so that a low composition drift is expected during polymerization. The instantaneous chemical composition of the copolymer is expected close to the overall composition of the monomer mixture and the copolymer has a random distribution of the repeat units. 
Therefore, the MAA repeat units are predicted being linked at random to EGDMA, grafted methacrylate and MAA units. The chemical composition of the final grafted polymer layer of Sil-MAA/MIP estimated from elemental analyses and TGA assuming that was no composition drift, was $2.3 \mathrm{mmol} \cdot \mathrm{g}^{-1}$ of MAA, $2.3 \mathrm{mmol} \cdot \mathrm{g}^{-1}$ of EGDMA and $1.1 \mathrm{mmol} \cdot \mathrm{g}^{-1}$ of grafted methacrylate. The grafted methacrylate units are considered as additional cross-links as they are attached to the silica surface. The present MAA/(EGDMA $+\gamma$-MPTS) mole ratio is 0.7 . Owing to such low content of MAA, there are mainly one or two MAA units in between two cross-links; and consequently, the probability for having longer sequences of poly(methacrylic acid) is low.

The $r_{\text {BuMA-MA }}$ value higher than one, indicates that the monomers EGDMA and surface bound methacryloyl esters, represented by BuMA, tend to undergo homopolymerization better than copolymerization with MA. The low value of $r_{\text {MA-BuMA, indicates that once a }}$ MA unit has been attached to the growing macroradical, the $\mathrm{MA}^{\bullet}$ terminal radical reacts better with BuMA than with MA. Therefore, the MA is mainly present as isolated units surrounded by its co-monomer units and its incorporation in the polymer material is low. Since copolymerization of MA is low, pure MA monomers remain in the solution once EGDMA and surface bound methacryloyl esters have been fully converted into polymer; and poly(maleic acid) homopolymer is formed in solution at the end of the polymerization process. This soluble poly(maleic acid) is eliminated during the subsequent washing processes.

The kinetic polymerizations of MA and EGDMA and surface bound methacryloyl esters (represented by BuMA in the calculations) were calculated as a function of overall conversion from classical theory of radical copolymerization in homogeneous solution [35] and using the reactivity ratios for the MA-BuMA pair (Figure 4). The conversion of the monomers calculated as a function of the conversion (Figure 4 left side) shows the 
much faster polymerization of BuMA compared to that of MA and its depletion from the solution at the end of polymerization, leaving there almost pure MA for homopolymerization. The instantaneous copolymers (Figure 4 right side) formed by copolymerization contained a low fraction of the poorly reactive MA at the beginning of polymerization. Conversely, the polymers formed at high conversion were rich in MA because the faster polymerization of BuMA made the reactional medium richer in MA; the polymer formed at the end was close to a poly(MA) homopolymer. The average composition of the copolymer followed the same trends as the instantaneous composition; of course it obviously reached the starting composition of the monomer mixture at full conversion. The copolymer grafted on silica was formed at low conversion; it contained a low amount of MA. As a more specific account, the mole fraction of MA in the copolymer at $30-40 \%$ conversion was less than half that of the starting monomer mixture. The same calculations for MAA instead of MA (Figure 5) showed the features of the formation of a random copolymer: the polymerization of MAA and methacrylic esters took place at the same rate and the chemical compositions of the copolymers were close to that of the starting monomer mixture. The distribution lengths of the poly(methacrylic acid) sequences (numbers of consecutive MAA units) show that $50 \%$ of the polymer sequences contained 2 MAA units or longer (Figure 6).

As an outcome of the considerations pertaining to copolymerization kinetics, the low reactivity of MA in the copolymerization with methacrylic esters results in its low incorporation into the grafted layer and its occurrence as isolated units in the copolymer. EGDMA can acts as cross-linking agent and as co-monomer also with the surface bound methacrylate, leading to formation of a highly cross-linked matrix entrapping isolated MA units. Methacrylic acid and methacrylic esters having similar reactivities, the copolymers have a random distribution of its MAA and cross-linking units, so that they 
are richer in MAA and they contain both isolated MAA units and short segments of poly(MAA). The MAA units are more abundant, but they are more mobile because of the lesser cross-linking density. While the simple reactivity of the monomer in homopolymerization gives a first indication, considering the reactivities in copolymerization provides much more useful insights. In particular, the chemical composition of the grafted layer departs from that of the monomers mixture when the reactivities of the co-monomers are different.
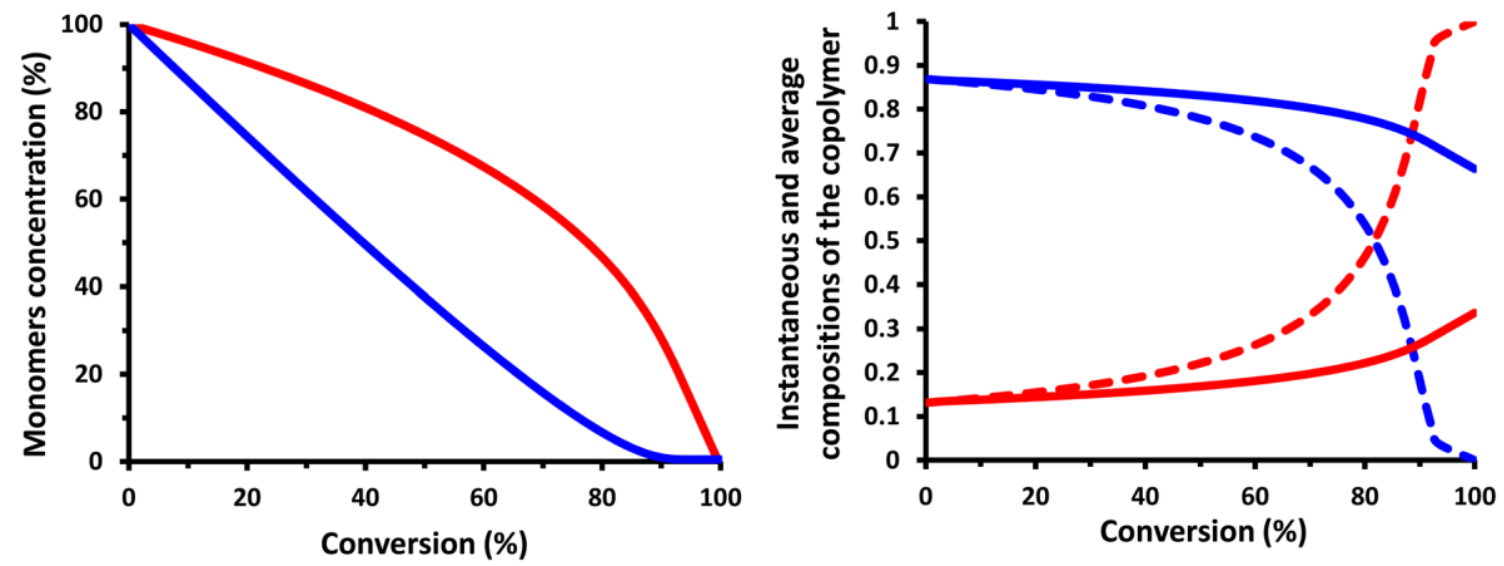

Figure 4. Kinetics of copolymerization of MA (red) and BuMA (blue). Left: concentration of residual monomers (relative to the starting concentration) along the conversion. Right: variations of the instantaneous (dotted lines) and average (solid lines) compositions of the formed copolymers against the conversion.
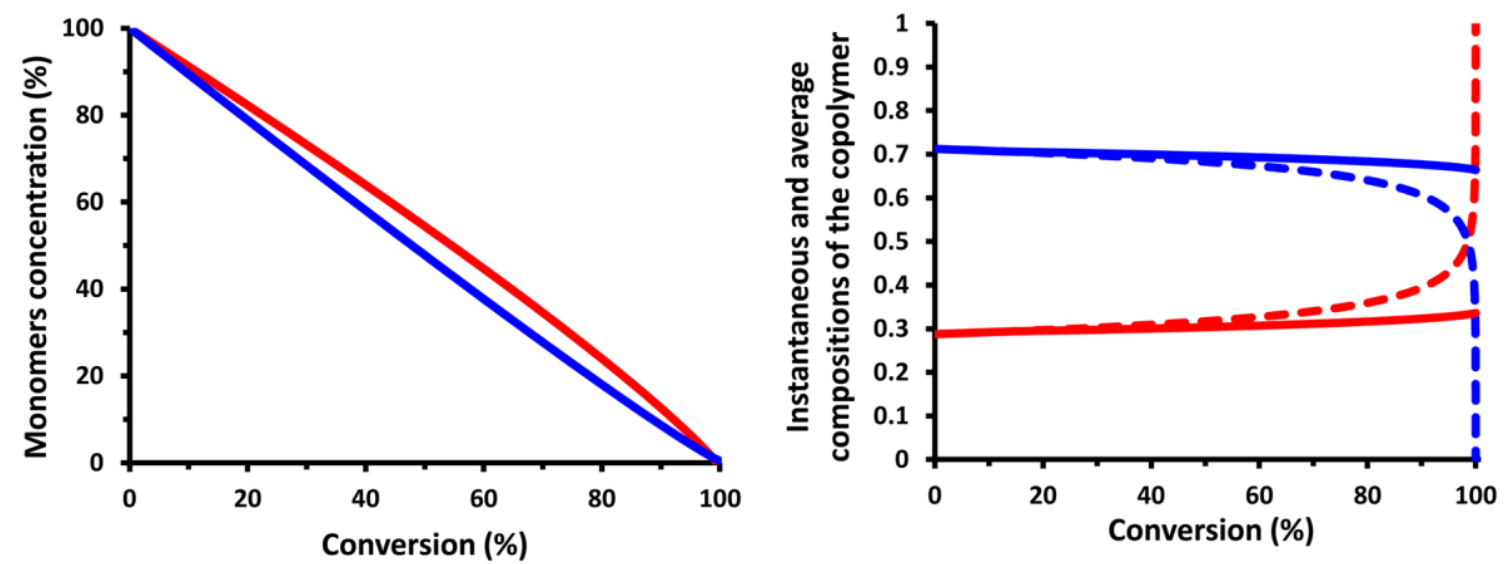

Figure 5. Kinetics of copolymerization of MAA (red) and BuMA (blue) presented as in Figure 4. 


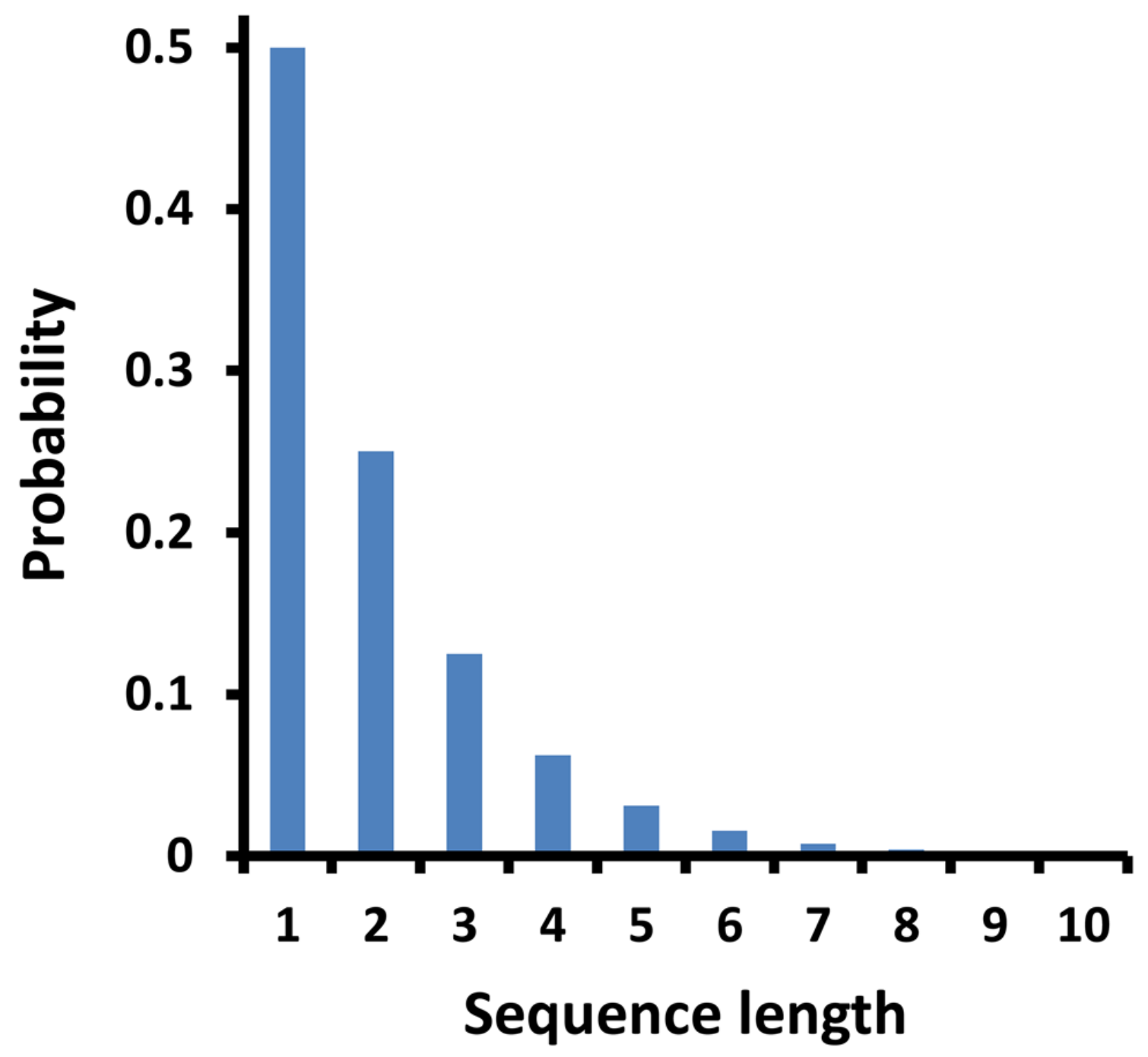

Figure 6. Distribution of sequence lengths of poly(MAA) for a random copolymer of average composition MAA:EGDMA = 50:50.

The consequences of such findings on the selectivity of adsorption of the MIPs are interesting since they contradict in some aspects the first intuitive ideas. The selectivity discussed in the present work refers to a comparison between the binding behavior of the target molecule to MIPs and NIPs. In this framework, selectivity comes from an excess number of binding sites in the MIPs. Let us notice that some authors based their discussion on selectivity defined as the differential binding of the target and interfering molecules, which is a different concept of selectivity. The higher selectivity for patulin uptake of Sil-MA/MIP compared to Sil-MAA/MIP comes from a higher density of selective binding sites. However, both the amount of grafted polymer material and the 
fraction of functional monomer in the grafted copolymers were larger for Sil-MAA/MIP. The much larger density of acidic sites in Sil-MAA/MIP did not yield a larger excess binding of patulin with respect to the NIP. The key difference between Sil-MA/MIP and Sil-MAA/MIP is the composition of the copolymer that sets the cross-linking density. A high cross-linking density is required for quenching the acidic sites oriented towards the template in such a way that their position is retained after elimination of the template for keeping maximum interactions with the target molecules. A high cross-linking density comes from a large EGDMA fraction in the copolymer composition, therefore, a low content of functional monomer. It might be considered that high cross-linking is at the expense of the density of binding sites. The present work shows that such poor density is largely compensated by the effects of the higher cross-linking density that prevent the loss of specific binding site when the material is too soft or when the functional monomers are incorporated into flexible polymer segments in between cross-links. Accordingly, the reactivity ratios of MA are favorable, resulting in the formation of isolated MA units surrounded by two cross-links. It is also worth to point out that adsorption of the target molecules takes place at the surface of the materials. Therefore, it is not useful to have a large amount of polymer material bound to the solid support as a thick coating because most of the functional units would be buried inside the material and not accessible for adsorption. A low density of functional units is enough for the formation of a large surface density at the external surface of the materials. Sil-MAA/MIP was coated with a thick layer of polymer that filled the interstices in between the silica particles, resulting in a loss of specific area as measured by the BET method. On the contrary, Sil-MA/MIP retained a large specific area and the thin coating was highly cross-linked at the adsorption sites. 
The predictions of the composition drift coming from reactivity ratios allowed a qualitative inference of experimental properties of the final material. This approach can be used as a guide to experimentalists regarding the choice of the monomers. A validation by comparison with experimental data would require chemical analyses of both the silicabound and free fractions of copolymer along a kinetic study. This will be the topic of future work.

As an outcome of the present work, the guidelines are as follows:

- The monomer grafted at the surface of silica should have high reactivity for it is copolymerized at the beginning of copolymerization;

- The functional monomer should have low reactivity for it is incorporated in the MIP material at low rate and the molecular imprints are isolated, so that the selectivity is high but the binding capacity is low;

- Low capacity should be compensated by a large specific area of the silica support.

\section{Conclusion}

This work highlights the effect of the reactivity of the functional monomer towards the cross-linking agent and the structure of the resulting molecular imprinted polymer in terms of distribution of the monomer in the polymer network and the rigidity of the formed matrix. The prepared MIPs having either methacrylic acid (Sil-MAA/MIP) or maleic acid (Sil-MA/MIP) as functional monomer, displayed quite different behavior both regarding their analytical performances and their morphological characteristics. It was shown that the difference in reactivity ratios resulted in a difference both in the distribution of functional monomers along the copolymer and in the crosslink density of the entire polymer matrix. The higher selectivity for patulin uptake observed with SilMA/MIP compared to Sil-MAA/MIP, is related to the more rigid polymer structure of 
the former that have allowed the retention of the shape of the binding sites despite the low proportion of the MA functional monomers incorporated in the polymer matrix.

\section{Acknowledgements}

This work was supported by funding from the "Partenariat Hubert Curien Utique" for French-Tunisian cooperation, grant $\mathrm{n}^{\circ} 19 \mathrm{G} 1204$.

\section{References}

[1] Sellergren B (2001) Molecularly Imprinted Polymers. Man-made mimics of antibodies and their applications in analytical chemistry. Elsevier, Amsterdam.

[2] Haupt K, Mosbach K (1998) Plastic antibodies: developments and applications. Trends Biotechnol 16: 468-475.

[3] Alvarez-Lorenzo C, Concheiro A (2013) Handbook of Molecularly Imprinted Polymers. Smithers Rapra, Shawbury UK.

[4] Canfarotta F, Waters A, Sadler R, McGill P, Guerreiro A, Papkovsky D, Haupt K, Piletsky S (2016) Biocompatibility and internalization of molecularly imprinted nanoparticles. Nano Res 9:3463-3477.

[5] Ayadi C, Anene A, Kalfat R, Chevalier Y, Hbaieb S (2019) Molecularly imprinted polyaniline on silica support for the selective adsorption of benzophenone-4 from aqueous media. Colloids Surfaces A 567:32-42.

[6] Arfaoui F, Khlifi A, Bargaoui M, Khalfaoui M, Kalfat R (2018) Thin Melamine Imprinted Sol Gel Coating on Silica Beads: Experimental and Statistical Physics Study. Chemistry Africa 1:175-185.

[7] Chianella I, Lotierzo M, Piletsky SA, Tothill IE, Chen B, Karim K, Turner APF (2002) Rational Design of a Polymer Specific for Microcystin-LR Using a Computational Approach. Anal Chem 74:1288-1293.

[8] Karlsson BCG, O’Mahony J, Karlsson JG, Bengtsson H, Eriksson LA, Nicholls IA (2009) Structure and Dynamics of Monomer-Template Complexation: An Explanation for Molecularly Imprinted Polymer Recognition Site Heterogeneity. J Am Chem Soc 131:13297-13304. 
[9] Cowen T, Stefanucci E, Piletska E, Marrazza G, Canfarotta F, Piletsky SA (2020) Synthetic Mechanism of Molecular Imprinting at the Solid Phase. Macromolecules 53:1435-1442.

[10] Anene A, Kalfat R, Chevalier Y, Hbaieb S (2016) Molecularly imprinted polymerbased materials as thin films on silica supports for efficient adsorption of Patulin. Colloid Surface A 497:293-303.

[11] Anene A, Hosni K, Chevalier Y, Kalfat R, Hbaieb S (2016) Molecularly imprinted polymer for extraction of Patulin in apple juice samples. Food Control 70:90-95.

[12] Puel O, Galtier P, Oswald IP (2010) Biosynthesis and toxicological effects of patulin. Toxins 2:613-631.

[13] Wright SA (2015) Patulin in food Curr Opin Food Sci 2:105-109.

[14] Tannous J, Atoui A, El Khoury A, Francis Z, Oswald IP, Puel O, Lteif R (2016) A study on the physicochemical parameters for Penicillium expansum growth and patulin production: effect of temperature, $\mathrm{pH}$, and water activity. Food Sci Nutrition 4:611-622.

[15] Anene A, Hosni K, Chevalier Y, Kalfat R, Hbaieb S (2017) Optimization of solid phase extraction based on molecularly imprinted polymer for patulin determination. J Tun Chem Soc 19:187-192.

[16] Thommes M, Kaneko K, Neimark AV, Olivier JP, Rodriguez-Reinoso F, Rouquerol J, Sing KSW (2015) Physisorption of gases, with special reference to the evaluation of surface area and pore size distribution (IUPAC Technical Report). Pure Appl Chem 87:1051-1069.

[17] Ktari N, Fourati N, Zerrouki C, Ruan M, Seydou M, Barbaut F, Nal F, Yaakoubi N, Chehimi MM, Kalfat R (2015) Design of a polypyrrole MIP-SAW sensor for selective detection of flumequine in aqueous media. Correlation between experimental results and DFT calculations. RSC Adv 5:88666-88674.

[18] Cormack PAG, Zurutuza Elorza A (2004) Molecularly imprinted polymers: synthesis and characterisation. J Chromatogr B 804:173-182.

[19] Vasapollo G, Del Sole R, Mergola L, Lazzoi MR, Scardino A, Scorrano S, Mele G (2011) Molecularly imprinted polymers: Present and future prospective. Int J Mol Sci 12:5908-5945.

[20] Fu X, Yang Q, Zhou Q, Lin Q, Wang C (2015) Template-monomer interaction in molecular imprinting: Is the strongest the best? Open J Org Polym Mater 5:58-68. 
[21] Zeynab T, Soleimani M, Mohammad M, Nikje A (2019) Characterization and performance evaluation of functional monomer effect on molecular imprinted polyurethane foam. J. Chromatogr A 1602:30-40

[22] Davies MP, De Biasi V, Perrett D (2004) Approaches to the rational design of molecularly imprinted polymers. Anal Chim Acta 504:7-14.

[23] Del Sole R, De Luca A, Catalano M, Mele G, Vasapollo G (2007) Noncovalent imprinted microspheres: preparation, evaluation and selectivity of DBU template. J Appl Polym Sci 105:2190-2197.

[24] Manning GS, Holtzer A (1973) Application of polyelectrolyte limiting laws to potentiometric titration. J Phys Chem 77:2206-2212.

[25] Dubin PL, Strauss UP (1970) Hydrophobic bonding in alternating copolymers of maleic acid. J Phys Chem 74:2842-2847.

[26] Dubin PL, Strauss UP (1967) Hydrophobic hypercoiling in copolymers of maleic acid and alkyl vinyl ethers. J Phys Chem 71:2757-2759.

[27] Sauvage E, Amos DA, Antalek B, Schroeder KM, Tan JS, Plucktaveesak N, Colby RH (2004) Amphiphilic maleic acid-containing alternating copolymers-1 Dissociation behavior and compositions. J Polym Sci Part B Polym Phys 42:3571-3583.

[28] Delben F, Paoletti S, Porasso RD, Benegas JC (2006) Potentiometric titrations of maleic acid copolymers in dilute aqueous solution: experimental results and theoretical interpretation. Macromol Chem Phys 207:2299-2310.

[29] Borukhov I, Andelman D, Borrega R, Cloitre M, Leibler L, Orland H (2000) Polyelectrolyte titration: Theory and experiment. J Phys Chem B 104:1102711034.

[30] Navarro-Villoslada F, San Vicente B, Moreno-Bondi MC (2004) Application of multivariate analysis to the screening of molecularly imprinted polymers for bisphenol A. Anal Chim Acta 504:149-162.

[31] Greenley RZ (1980) Recalculation of some reactivity ratios. J Macromol Sci Chem A $14: 445-515$.

[32] Świtała-Żeliazkow M (1999) Radical copolymerization of maleic acid with styrene. Eur Polym J 35:83-88. 
[33] Greenley RZ (1999) $Q$ and $e$ values for free radical copolymerizations of vinyl monomers and telogens. In: Brandrup J, Immergut EH, Grulke EA (ed) Polymer Handbook, $4^{\text {th }}$ edn. Wiley, New York, Chap II, pp 309-320.

[34] Paxton TR (1963). Copolymerization reactivity ratios acrylic and methacrylic acids with butyl acrylate and butyl methacrylate. J Polym Sci B Polym Lett 1:7376.

[35] Odian G (2004) Principles of Polymerization. In: Hoboken NJ (ed) Wiley, New York, Chap 6, pp 464-484. 